EXTENDED REPORT

\title{
Subthreshold diode micropulse photocoagulation for the treatment of clinically significant diabetic macular oedema
}

\author{
J K Luttrull, D C Musch, M A Mainster
}

Br J Ophthalmol 2005;89:74-80. doi: 10.1136/bjo.2004.051540

\begin{abstract}
Aim: To report the visual and clinical outcomes of a pilot study of subthreshold diode micropulse (SDM) laser photocoagulation for clinically significant diabetic macular oedema (CSMO).

Methods: The results of infrared $(810 \mathrm{~nm})$ SDM laser photocoagulation for CSMO were retrospectively reviewed in 95 eyes of 69 consecutive patients with mild to moderate non-proliferative diabetic retinopathy. The same laser parameters were used for each patient. Only the number of laser applications varied between patients, depending on their macular findings. Primary outcome measures were Snellen visual acuity, fluorescein angiographic leakage, and CSMO status. Results: Visual acuity was stable or improved in $85 \%$ of treated eyes, with a mean follow up of 12.2 months (range 3-29 months). CSMO decreased in $96 \%$ and resolved in $79 \%$ of treated eyes. No adverse laser events occurred. No laser lesions were detectable ophthalmoscopically or angiographically after treatment, consistent with calculations based on ANSI Z136.1 laser safety standards suggestive of only histologically detectable tissue effects at the laser exposure levels. No laser scarring was observed during the follow up period.

Conclusion: Subthreshold diode micropulse laser photocoagulation minimises chorioretinal damage in the management of CSMO and demonstrates a beneficial effect on visual acuity and CSMO resolution. Prospective studies are needed to fully evaluate this technique.
\end{abstract}

See end of article for authors' affiliations

Correspondence to: Jeffrey K Luttrull, MD, 3160 Telegraph Road, Suite 230, Ventura, CA, 93003, USA; jkluttrull@aol.com Accepted for publication 3 June 2004

C omplications of diabetic retinopathy remain a leading cause of vision loss in people under 60 years of age..$^{1-4}$ Diabetic macular oedema is the most common cause of legal blindness in this patient group. ${ }^{35}{ }^{6}$ The Early Treatment of Diabetic Retinopathy Study (ETDRS) showed that visible end point focal laser photocoagulation reduces the risk of vision loss in patients with clinically significant diabetic macular oedema (CSMO). ${ }^{7}$ This conventional photocoagulation protocol is effective for treating CSMO, but it causes visible laser scars that can enlarge postoperatively, ${ }^{8-10}$ and complications that include choroidal neovascularisation $(\mathrm{CNV}),{ }^{11}{ }^{12}$ subretinal fibrosis ${ }^{13}{ }^{14}$ and visual field loss. ${ }^{15-20}$

Intrinsic damage from visible end point laser photocoagulation has prompted interest in developing alternative methods for treating CSMO such as intravitreal steroid injection $^{21-23}$ and vitrectomy, ${ }^{24-27}$ but laser photocoagulation damage itself can be decreased by reducing laser exposure duration and using a subvisible clinical end point for therapy. ${ }^{20}{ }^{28-34}$ We used both of these approaches in a subthreshold diode micropulse (SDM) laser photocoagulation protocol that one of us (JKL) employed to treat 69 consecutive patients with CSMO in an effort to minimise laser induced chorioretinal damage.

\section{PATIENTS AND METHODS}

A retrospective review of the records of all patients undergoing infrared $(810 \mathrm{~nm})$ SDM laser photocoagulation for retinal vascular disease in a solo private vitreoretinal subspecialty practice was performed. For the time period between January 2000 and February 2003, 231 patients (348 eyes) were treated with SDM and had at least 3 months of postoperative follow up. Patients with severe nonproliferative and proliferative diabetic retinopathy, as well as those with other visually significant ocular diseases, such as retinal vascular occlusion, age related macular degeneration, and advanced or uncontrolled glaucoma, were excluded from review, yielding 95 consecutive eyes of 69 patients with mild to moderate non-proliferative diabetic retinopathy undergoing SDM laser photocoagulation treatment for CSMO. Each treated patient received and signed an informed consent including discussion of potential adverse effects, possible treatment failure, and possible need for additional or other treatment.

Recorded data included patient age, sex, race, type and duration of diabetes mellitus, and other systemic disease. For the treated eye, we documented ocular fundus pigmentation, lens status, ocular disease other than diabetic retinopathy, previous laser treatment or vitreous surgery, type and severity of diabetic retinopathy, presence of epiretinal membrane or posterior hyaloidal contraction, intraocular pressure, and Snellen visual acuity. All visual acuities were assessed by a single technician who used a uniform testing procedure. CSMO was graded in order of severity as focal ("category l"), diffuse (involving all four quadrants of macula including fovea) ("category 2"), cystoid foveal oedema/degeneration ("category 3"), and macular ischaemia ("category 4") (tables 1 and 2)

The diagnosis of CSMO was made according to ETDRS criteria, by dilated slit lamp macular biomicroscopy. Intravenous fundus fluorescein angiography was performed to confirm and characterise microvascular leakage. Following informed consent, SDM laser photocoagulation was performed. Topical anaesthesia was administered and a $0.96 \times$ inverted image ophthalmoscopic contact lens was applied to the cornea. All areas of ophthalmoscopically detectable macular oedema and angiographically apparent retinal

\footnotetext{
Abbreviations: CNV, choroidal neovascularisation; CSMO, clinically significant diabetic macular oedema; ETDRS, Early Treatment of Diabetic Retinopathy Study; HSP, heat shock protein; MPE, maximum permissible exposure; PEDF, pigment epithelium derived factor; RPE, retinal pigment epithelium; SDM, subthreshold diode micropulse; VEGF, vascular endothelial growth factor
} 
Table 1 Visual acuity change in logMAR units by CSMO status at baseline

\begin{tabular}{lllll}
\hline CSMO status & 1st VA mean & Last VA mean & Mean difference (SD) & $\mathbf{p}$ Value $(\boldsymbol{t}$ test) \\
\hline Category $1(n=72)$ & 0.30 & 0.38 & $+0.09(0.28)$ & 0.0077 \\
Category 2 $(n=17)$ & 0.54 & 0.48 & $-0.05(0.22)$ & 0.33 \\
Category 3 $(n=5)$ & 0.62 & 0.42 & $-0.20(0.20)$ & 0.09 \\
Category 4 $(n=1)$ & 1.30 & 0.60 & $-0.70(N A)$ & NA \\
Overall $(n=95$ eyes) & 0.37 & 0.41 & $+0.04(0.28)$ & 0.17 \\
\hline
\end{tabular}

$\mathrm{CSMO}=$ clinically significant diabetic macular oedema. $\mathrm{VA}=$ visual acuity. $\mathrm{NA}=$ not applicable. * Significant difference in mean VA change by CSMO status $(p=0.0016$, ANOVA).

\begin{tabular}{|c|c|c|c|}
\hline CSMO status & $\%$ gain $3+$ lines & $\% \pm<3$ lines & $\%$ lose $3+$ lines \\
\hline Category $1(n=72)$ & $5.6 \%(4)$ & $76.4 \%(55)$ & $18.1 \%(13)$ \\
\hline Category $2(n=17)$ & $11.8 \%(2)$ & $82.4 \%(14)$ & $5.9 \%(1)$ \\
\hline Category $3(n=5)$ & $20.0 \%(1)$ & $80.0 \%(4)$ & $0.0 \%(0)$ \\
\hline Category $4(n=1)$ & $100 \%(1)$ & - & - \\
\hline Overall ( $n=95$ eyes) & $8.4 \%(8)$ & $76.8 \%(73)$ & $14.7 \%(14)$ \\
\hline
\end{tabular}

vascular leakage were treated with confluent laser applications, up to the edge of the foveal avascular zone if it was thickened. The laser treatment parameters used for each patient were $125 \mu \mathrm{m}$ laser spot diameter, 0.3 second laser exposure (envelope of micropulses), and $750 \mathrm{~mW}$ of $810 \mathrm{~nm}$ infrared diode laser radiation (Iris Medical OcuLight SLX photocoagulator, Iridex Corporation, Mountain View, CA, USA). Each laser exposure consisted of 150, $100 \mu$ s micropulses, delivered every $2 \mathrm{~ms}(100 \mu \mathrm{s}$ ON, $1900 \mu \mathrm{s}$ OFF, $500 \mathrm{~Hz}, 5 \%$ duty factor). Only the number of laser spots varied between patients, according to the extent of their CSMO. Since photocoagulation lesions were not ophthalmoscopically apparent, treated areas were often retreated ("painted") during each treatment session to ensure complete coverage.

Treated patients were followed at 3 month intervals postoperatively. At each follow up examination, the records were reviewed for the following data: best corrected Snellen visual acuity, diabetic retinopathy status, and macular oedema status. CSMO was recorded as resolved, improved, worsened, or new/recurrent. In patients for whom retreatment was indicated or considered, fundus photography and intravenous fundus fluorescein angiography were repeated. Fluorescein angiographic leakage was recorded as resolved, improved, worsened, or new/recurrent. For patients undergoing re-treatment, the technique described above was repeated and all laser parameters were recorded. Conventional laser photocoagulation was available for patients unresponsive to SDM photocoagulation, but not deemed necessary for any of the study patients.

Patient data obtained from retrospective chart review were incorporated into a computer database. Statistical analyses made use of paired, Student's $t$ tests for before/after treatment comparisons, analysis of variance for comparing means, and $\chi^{2}$ testing for comparing percentages. The unit of analysis was eyes. p Values less than or equal to 0.05 were considered significant.

\section{RESULTS}

In all, 95 eyes of 69 patients, aged 42-90 years (mean 69 years) were treated. Postoperative follow up ranged from 3-29 months (mean 12.2 months). A total of 43 patients were female and 26 were male. 49 were white, 16 Hispanic, and four of Asian descent; 33 had systemic hypertension and 39 were insulin dependent. Treatment episodes ranged from one to six per eye (mean 1.7). The total number of SDM laser exposures (envelopes) per eye ranged from 27 to 3427 (mean 543).

\section{Postoperative visual acuity}

By $\log$ MAR unit analysis, overall postoperative visual acuity remained essentially stable relative to the preoperative visual acuity level (an overall change of +0.14 logMAR units, table 1). As the preoperative CSMO status worsened, however, the mean change in logMAR scores from preoperative levels increased significantly, reflecting more visual acuity loss in eyes with a poorer preoperative CSMO state. Preoperative visual acuity (VA) level significantly influenced treatment benefit $(p=0.0016$, analysis of variance). While perhaps reflective of a floor effect, the 45 eyes with good or fair preoperative VA demonstrated an increased logMAR score (worsened VA) after treatment of about 0.1 units, whereas 50 eyes with poor preoperative VA remained stable (mean change of $+0.02 \log$ MAR units, table 3 ). Patients with progressively worsening fluorescein angiographic leakage postoperatively tended toward poorer visual outcomes (table 4). A similar association appears in patients with progressively worsening macular oedema despite SDM treatment (tables 5-7). Conversely, patients with improved

Table 3 Visual acuity change in logMAR units by initial VA status

\begin{tabular}{|c|c|c|c|c|}
\hline Initial VA status & 1st VA mean & Last VA mean & Mean difference (SD)* & $p$ Value ( $t$ test) \\
\hline $\begin{array}{l}\text { Good }(n=7) \\
\text { Fair }(n=38) \\
\text { Poor }(n=50) \\
\text { Overall }(n=95 \text { eyes })\end{array}$ & $\begin{array}{l}0.00 \\
0.16 \\
0.58 \\
0.37\end{array}$ & $\begin{array}{l}0.12 \\
0.26 \\
0.56 \\
0.41\end{array}$ & $\begin{array}{l}+0.12(0.15) \\
+0.11(0.27) \\
-0.02(0.30) \\
+0.04(0.28)\end{array}$ & $\begin{array}{l}0.07 \\
0.02 \\
0.62 \\
0.17\end{array}$ \\
\hline
\end{tabular}


Table 4 Visual acuity change: progressive leakage versus some (reduced) leakage versus resolved leakage

\begin{tabular}{lllll}
\hline FA leak status & 1st VA mean & Last VA mean & Mean difference (SD)* & p Value $(\boldsymbol{t}$ test) \\
\hline Progressive $(n=3)$ & 0.27 & 0.73 & $+0.46(0.91)$ & 0.47 \\
Some present $(n=25)$ & 0.42 & 0.48 & $+0.06(0.29)$ & 0.30 \\
Resolved $(n=67)$ & 0.35 & 0.36 & $+0.01(0.22)$ & 0.63 \\
Overall $(n=95$ eyes) & 0.37 & 0.41 & $+0.04(0.28)$ & 0.17 \\
\hline \multirow{2}{*}{ *Significant difference in mean VA change by FA leakage status after follow up ( $p=0.0234$, ANOVA). } &
\end{tabular}

Table 5 Visual acuity change: progressive leakage versus some (reduced) leakage versus resolved leakage

\begin{tabular}{llll}
\hline AFA leak status & $\%$ gain 3+ lines & $\% \pm<3$ lines & $\%$ lose 3+ lines \\
\hline Progressive $(n=3)$ & $0.0 \%(n=0)$ & $66.7 \%(n=2)$ & $33.3 \%(n=1)$ \\
Some present $(n=25)$ & $12.0 \%(n=3)$ & $64.0 \%(n=16)$ & $24.0 \%(n=6)$ \\
Resolved $(n=67)$ & $7.5 \%(n=5)$ & $82.1 \%(n=55)$ & $10.4 \%(n=7)$ \\
Overall $(n=95$ eyes) & $8.4 \%(n=8)$ & $76.8 \%(n=73)$ & $14.7 \%(n=14)$ \\
\hline
\end{tabular}

or resolved fluorescein angiographic leakage or macular oedema had stable visual acuity postoperatively.

Outcome assessment by change in lines of Snellen visual acuity postoperatively reveals a significant influence by preoperative CSMO subclass $\left(\mathrm{p}=0.02, \chi^{2}\right.$ test $)$, with overall postoperative visual acuity unchanged (plus or minus two Snellen lines) in $76.8 \%$, worsened by three or more lines in $14.7 \%$, and improved in $8.4 \%$. By this measure, postoperative visual results in each subclass of CSMO appear inversely related to CSMO severity (table 1). Systemic hypertension status, preoperative visual acuity level, and postoperative fluorescein leakage status did not affect treatment benefit significantly by the same outcome measure, although the small number of patients with worsening angiographic leakage despite treatment appeared to trend unfavourably (table 4). Similar results by this outcome measure are demonstrated postoperatively for CSMO status, and 79\% of eyes exhibiting complete resolution of macular oedema postoperatively had significantly better visual outcomes compared to $17 \%$ of eyes with persistent and $4 \%$ of eyes with worsening macular oedema (table 8).
No patient in the study reported pain during laser surgery. No intraoperative or postoperative treatment complications were observed. Patients often reported subjective visual improvement within 24 hours of SDM treatment.

Postoperative intravenous fluorescein angiography and fundus photography were performed in all patients considered for additional SDM treatment. In addition, early in the course of using this technique, several patients underwent postoperative photography and intravenous fluorescein angiography as early as 1, 10, 30, and 60 minutes, and between 1 and 12 weeks post-treatment. Despite the large mean number of treatment applications per eye, at no point did any patient in this report exhibit any acute or late ophthalmoscopic or fluorescein angiographic evidence of laser treatment (figs 1-3).

\section{DISCUSSION}

Conventional visible end point laser photocoagulation reduces the short term and long term risk of vision loss from CSMO. ${ }^{735}$ Smaller studies have indicated that indirect

Table 6 Visual acuity change in eyes with worsened CSMO versus some or resolved CSMO on final examination

\begin{tabular}{lllll}
\hline CSMO status & 1st VA mean & Last VA mean & Mean difference (SD)* & $p$ Value $(t$ test) \\
\hline $\begin{array}{l}\text { Worsened }(n=4) \\
\text { Some/resolved }\end{array}$ & 0.49 & 1.08 & $+0.58(0.66)$ & 0.18 \\
$(n=91)$ & 0.38 & $+0.02(0.24)$ & 0.51 \\
Overall ( $n=95$ eyes) & 0.37 & 0.41 & $+0.04(0.28)$ & 0.17 \\
\hline
\end{tabular}

*Significant difference in mean VA change by CSMO status relative to baseline at the final examination $(p<0.0001$, ANOVA).

Table 7 Visual acuity change in eyes with worsened versus some present versus resolved CSMO on final examination

\begin{tabular}{lllll}
\hline CSMO status & 1st VA mean & Last VA mean & Mean difference (SD)* & $\mathbf{p}$ Value ( $\boldsymbol{t}$ test) \\
\hline Worsened $(n=4)$ & 0.49 & 1.08 & $+0.58(0.66)$ & 0.18 \\
Some present $(n=16)$ & 0.55 & 0.61 & $+0.06(0.35)$ & 0.53 \\
Resolved $(n=75)$ & 0.32 & 0.33 & $+0.01(0.21)$ & 0.75 \\
Overall $(n=95$ eyes) & 0.37 & 0.41 & $+0.04(0.28)$ & 0.17 \\
\hline *Significant difference in mean VA change by CSMO status relative to baseline at the final examination \\
( $p=0.0002$, ANOVA).
\end{tabular}


Table 8 Visual acuity change in eyes with worsened versus some (reduced) present versus resolved CSMO on final examination

\begin{tabular}{llll}
\hline CSMO status & \% gain 3+ lines & $\% \pm<3$ lines & $\%$ lose 3+ lines \\
\hline Worsened $(n=4)$ & $0.0 \%(n=0)$ & $50.0 \%(n=2)$ & $50.0 \%(n=2)$ \\
Some present $(n=16)$ & $12.5 \%(n=2)$ & $50.0 \%(n=8)$ & $37.5 \%(n=6)$ \\
Resolved $(n=75)$ & $8.0 \%(n=6)$ & $84.0 \%(n=63)$ & $8.0 \%(n=6)$ \\
Overall $(n=95)$ & $8.4 \%(n=8)$ & $76.8 \%(n=73)$ & $14.7 \%(n=14)$ \\
\hline
\end{tabular}

("grid") and less intense photocoagulation can also be effective in the treatment of CSMO. ${ }^{19} 31333436-45$

In visible end point photocoagulation, laser light absorption heats pigmented tissues at the laser site. Heat conduction spreads this temperature increase from the retinal pigment epithelium (RPE) and choroid to overlying nonpigmented and adjacent unexposed tissues. Laser lesions become visible immediately when damaged neural retina overlying the laser site loses its transparency and scatters white ophthalmoscopic light back towards the observer. There are different exposure thresholds for retinal lesions that are haemorrhagic, ophthalmoscopically apparent, or angiographically demonstrable. In clinical parlance, however, a "threshold" lesion is one that is barely visible ophthalmoscopically at treatment time, a "subthreshold" lesion is one that is not visible at treatment time, and "suprathreshold" laser therapy is retinal photocoagulation performed to a readily visible end point.

Decades of experimental data provide a basis for laser safety standards such as the American National Standards Institute (ANSI) Z136.1 standard, ${ }^{46}$ which specifies a maximum permissible exposure (MPE) level for a particular set of laser parameters. In experimental studies, a "threshold" laser exposure is usually the estimated dose of laser radiation needed for a $50 \%$ probability of a visible laser effect (that is, the ED-50). ${ }^{47}{ }^{48}$ The threshold for visible laser effects is roughly twice that of subvisible effects detectable only histologically. ${ }^{48}$ MPEs provide a variable safety margin for laser manufacturers and users, ranging roughly from $10 \times$ for $100 \mathrm{~ms}$ to $100 \times$ for $100 \mu \mathrm{s}$ exposures. ${ }^{49}$

The value of ANSI Z136.1 MPEs as benchmarks for various clinical treatment parameters is limited by their dependence on laser pulse duration and tissue pigmentation. For example, in terms of energy delivered to the cornea, clinical parameters are $1.4 \times$ MPE for verteporfin photodynamic therapy, ${ }^{50} 9.3 \times$ MPE for diode laser transpupillary thermotherapy for $\mathrm{CNV},{ }^{51}{ }^{52} 37 \times$ MPE for argon or FD-YAG green (300 mW, 0.2 second, $200 \mu \mathrm{m}$ spot diameter) conventional photocoagulation and more than $200 \times$ MPE for $0.8 \mu \mathrm{s}$ FD:YAG green $^{34}$ repetitive pulse photocoagulation. ${ }^{49}$ The SDM laser photocoagulation parameters used in this study ( $125 \mu \mathrm{m}$ diameter spot size, $750 \mathrm{~mW}, 0.3$ second laser exposure (envelope), $500 \mathrm{~Hz}, 100 \mu$ s micropulses) are $47 \times$ MPE, a level at which laser effects would be expected to be detectable histologically but not ophthalmoscopically or angiographically for $100 \mu$ s pulses.

In addition to using an end point not clinically visible, we also restricted laser photocoagulation damage by using micropulse (repetitive pulse) photocoagulation. Shortening a laser pulse limits the spread of photocoagulation damage caused by heat conduction during a laser exposure. Delivering all the energy needed for a conventional photocoagulation lesion in one very brief exposure, however, increases the risk of iatrogenic haemorrhage. ${ }^{20}$ Micropulse
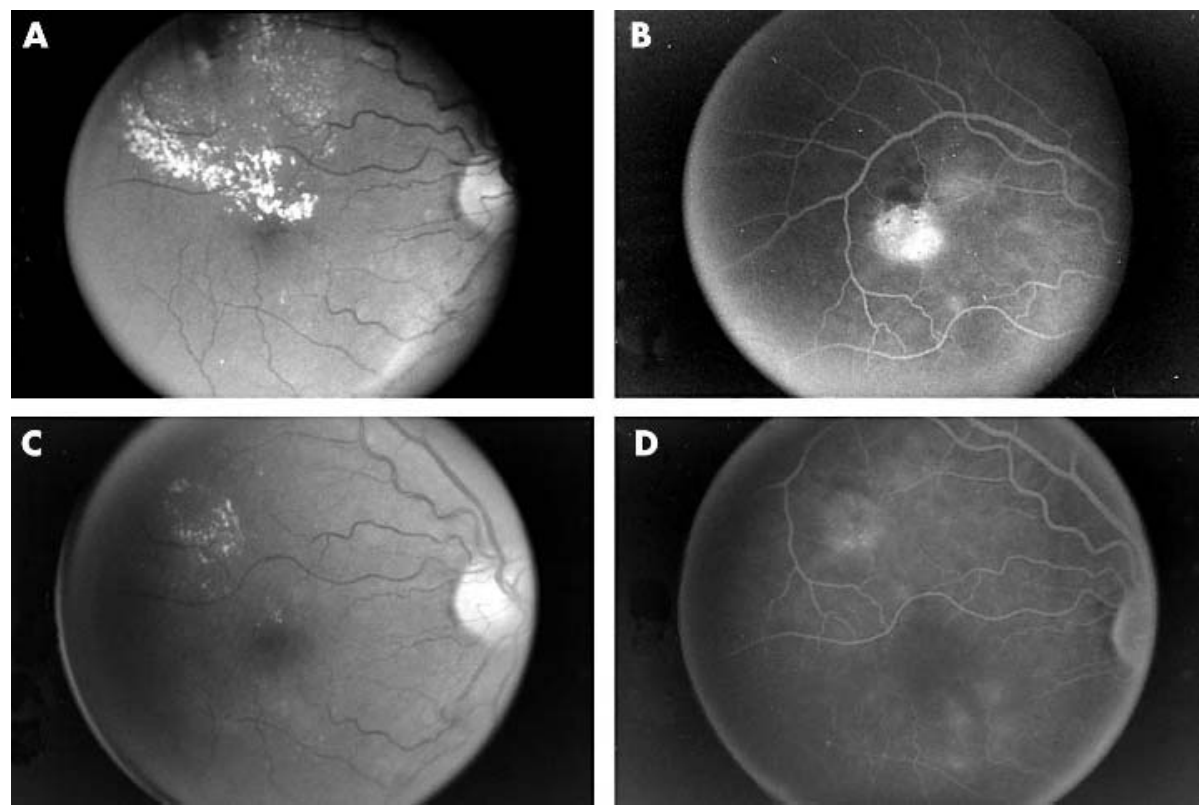

Figure 1 (A) Patient 206. Preoperative red-free fundus photograph demonstrating clinically significant diabetic macular oedema. (B) Preoperative intravenous fundus fluorescein angiogram. Note prominent focal retinal microvascular leakage. This patient was treated confluently throughout the area of leakage and retinal thickening with 269 applications of SDM photocoagulation. (C) Red-free fundus photograph 10 months following SDM photocoagulation. Note resolution of macular oedema and hard exudates. (D) Intravenous fundus fluorescein angiogram 10 months post-SDM macular photocoagulation. Note persistent but diminished focal microvascular leakage, and absence of angiographically visible pigment disturbance or chorioretinal scarring. 

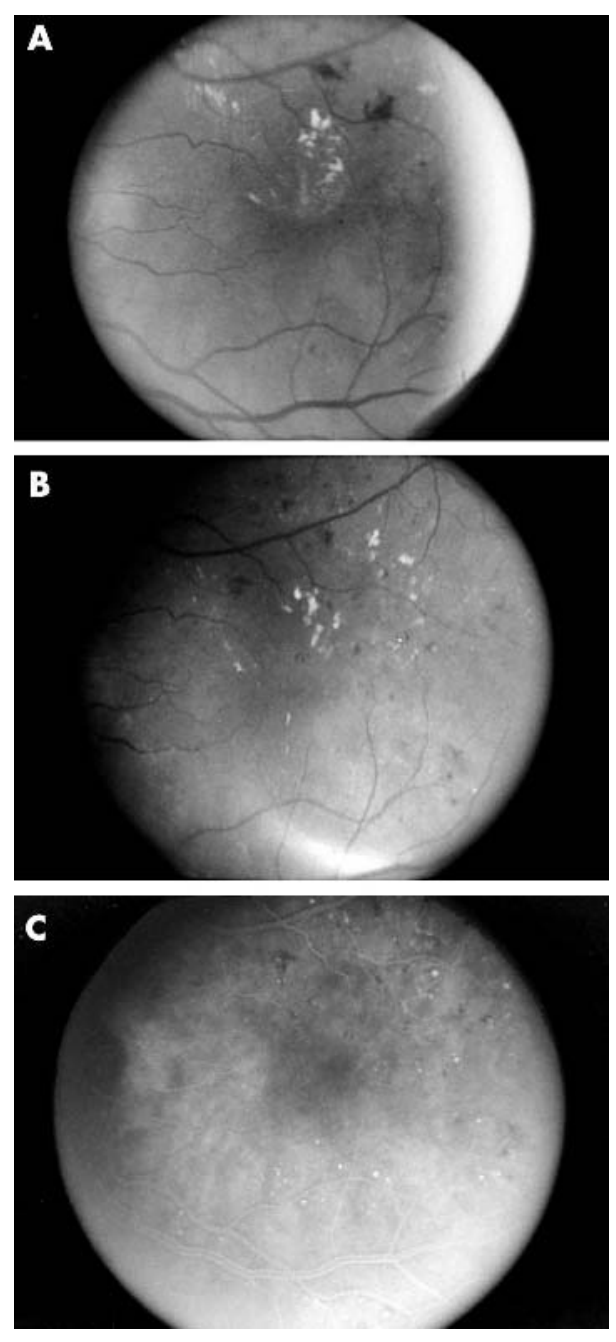

Figure 2 (A) Patient 141. Preoperoperative red-free fundus photograph demonstrating clinically significant diabetic macular oedema. (B) Red-free fundus photograph 5 months following 1663 applications of SDM photocoagulation applied confluently to the areas of biomicroscopically visible retinal thickening. The clinically significant macular oedema has resolved. (C) Postoperative intravenous fundus fluorescein angiogram. Note the absence of angiographically visible pigment disturabance or chorioretinal scarring following SDM macular photocoagulation.

photocoagulation avoids this problem by delivering the laser energy as a series of very brief micropulses within a single laser exposure (envelope). ${ }^{20} 28{ }^{30}$ Retinal temperature rise from each micropulse is lower than the temperature elevation that would occur if all the laser energy were delivered in a single micropulse. ${ }^{20}$

Each of the 0.3 second laser exposures (envelopes) consisted of 150 micropulses. Individual micropulses were $100 \mu \mathrm{s}$ in duration and delivered every $200 \mathrm{~ms}(500 \mathrm{~Hz}, 5 \%$ duty factor). Each micropulse denatures a small fraction of target tissue molecules, repetitive pulses add up to produce laser effects according to the $\mathrm{n}^{-1 / 4}$ law, ${ }^{204647}$ and experimental data show that each pulse causes some denaturation of intracellular proteins at exposures $1 / 10$ th to $1 / 100$ th of single pulse thresholds. ${ }^{29} 54$ During a $100 \mu \mathrm{s}$ micropulse, heat travels only about $4 \mu \mathrm{m}$, based on the standard assumption that the RPE and choroid have thermal properties similar to water. ${ }^{55}{ }^{56}$ Thus, our parameter selection limits heat conduction effects to RPE cells, which are roughly $10 \mu \mathrm{m}$ in height and width, ${ }^{58}$ and to immediately adjacent tissues such as the outermost portions of photoreceptor outer segments.
A variety of mechanisms may be involved in successful laser photocoagulation for CSMO, including (1) the upregulation or downregulation of chemical factors such as pigment epithelium derived factor (PEDF), vascular endothelial growth factor (VEGF) and heat shock protein (HSP), (2) thermal vascular thrombosis, sclerosis, or leucostasis, and (3) alterations in inner and outer blood-retinal barriers that can briefly increase and subsequently decrease retinal oedema. ${ }^{52}$ 59-67 Optimising photocoagulation awaits elucidation of its beneficial mechanisms, but the adverse effects of visible end point photocoagulation are caused by thermal damage to the RPE and adjacent cells. ${ }^{8-14} 6869$ Subthreshold photocoagulation protocols decrease this chorioretinal damage. ${ }^{19} 2031-3442444570$ As noted above, patients treated with SDM photocoagulation did not have ophthalmoscopically or angiographically detectable lesions.

In our study, visual acuity stabilised or improved in $85 \%$ of treated eyes, with oedema decreasing in $96 \%$ and resolving in $79 \%$ of treated eyes. In the $15 \%$ of eyes losing three or more Snellen lines of visual acuity, 10 of 14 eyes had improved or resolved CSMO. Cataract formation was the most common cause of this decreased visual acuity. No patient required supplemental conventional suprathreshold photocoagulation. No haemorrhage, sudden visual loss, or other adverse treatment effect was observed in any patient. No patient undergoing fundus fluorescein angiography within 1 month of SDM treatment demonstrated early postoperative worsening of CSMO or angiographic leakage. Fluorescein angiographic leakage often persisted despite resolution of CSMO and improvement in visual acuity, a finding also reported in the ETDRS. ${ }^{10}$ Patients with progressively worsening fluorescein angiographic leakage (three of 95 eyes) had worse visual acuity outcomes.

The SDM laser photocoagulation protocol we used has the weaknesses and strengths of other subthreshold laser protocols. There is no ophthalmoscopically apparent end point to help titrate or confirm therapy. Conversely, minimising chorioretinal laser damage permits confluent therapy and re-treatment of regions of macular oedema as demonstrated in our study. Treatment can be time consuming, but similar clinical outcomes might be achieved more rapidly by using 0.15 second rather than 0.3 second exposure envelopes, $300 \mu$ s rather than $100 \mu$ s micropulses (that is, a $15 \%$ rather than a $5 \%$ duty factor) and $800 \mathrm{~mW}$ rather than $750 \mathrm{~mW}$ of diode $810 \mathrm{~nm}$ infrared laser power. These alternative parameters provide a laser exposure that is $55 \times$ ANSI Z136.1 MPE, similar to the $47 \times$ MPE exposure used in this study.

Subthreshold diode micropulse photocoagulation is well tolerated by patients because it is painless and the infrared treatment beam is invisible. Areas of macular oedema can be treated aggressively because of reduced laser damage. SDM photocoagulation does not produce chorioretinal scars which could expand or increase the risk of iatrogenic choroidal neovascularisation. There is no angiographically demonstrable blood-retinal barrier breakdown, as in $0.7 \mu$ s subthreshold but angiographically apparent FD-YAG green repetitive pulse photocoagulation..$^{34}$ The reduced damage from SDM laser treatment may permit earlier intervention ${ }^{59}$ than current guidelines developed from the ETDRS, ${ }^{1072} 73$ perhaps improving long term visual prognosis.

The validity of this pilot study is limited by its small size and retrospective nature. We lacked uniform postoperative patient follow up and therefore grouped the last available visual acuity measures as the "last VA" for outcome assessment. None the less, we had at least 3 months of follow up for each patient, providing some confidence in this measure as a treatment outcome. Each eye was considered to be an independent unit for analysis purposes, which unduly 

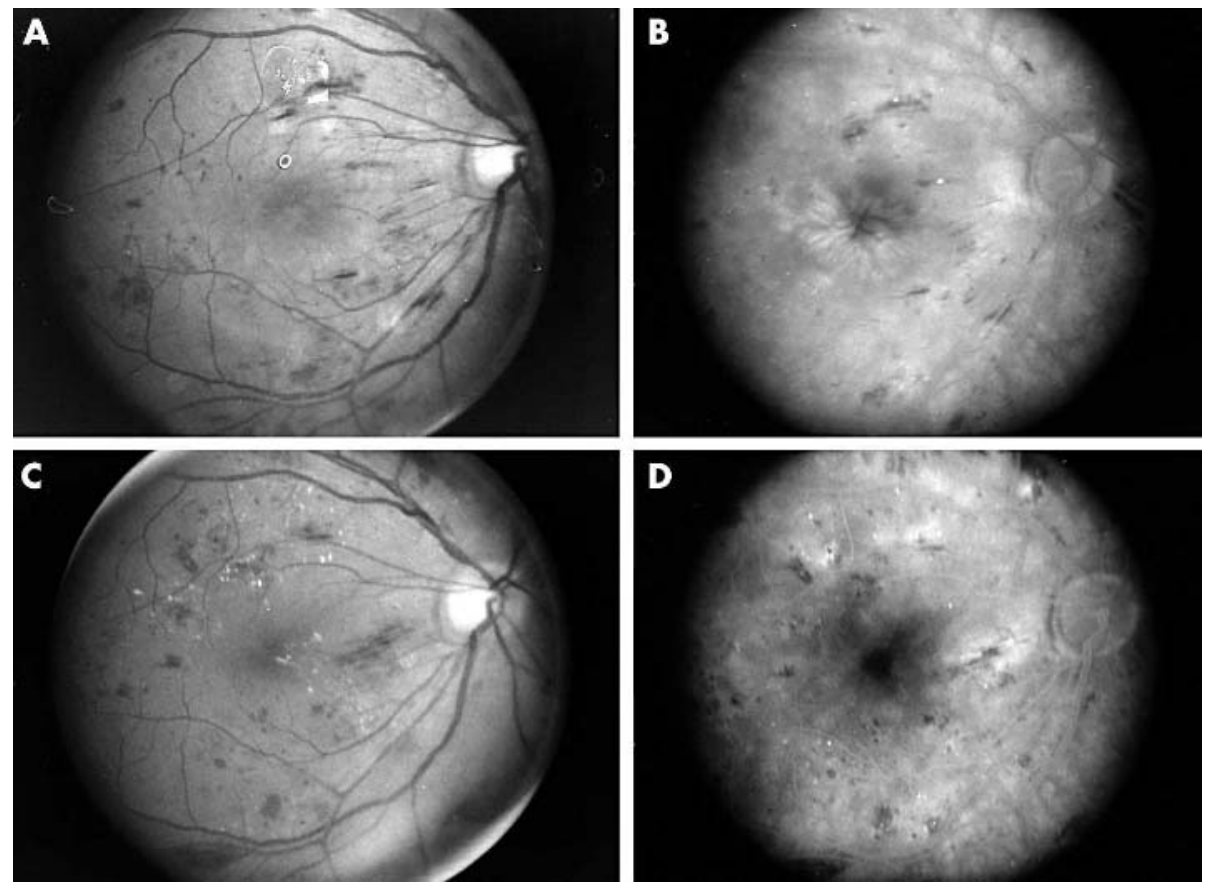

Figure 3 (A) Patient 107. Preoperative red-free fundus photograph of patient with diffuse clinically significant diabetic macular oedema and foveal cysts. (Note film development artefacts superior to fovea and at temporal edge of photograph.) (B) Late phase preoperative intravenous fundus fluorescein angiogram of diffuse clinically significant diabetic macular oedema. Note cystoid leakage pattern in fovea. This patient was treated with 602 applications of SDM photocoagulation in a nearly confluent grid pattern throughout the macula extending to the edge of the fovea circumferentially. (C) Red-free fundus photograph 8 months following SDM photocoagulation. Note marked reduction in macular oedema without visible chorioretinal scarring or pigmentary disturbance. (D) Postoperative intravenous fundus fluorescein angiogram. Note marked reduction in diffuse and cystoid leakage. Note absence of angiographically visible pigmentary disturbance or chorioretinal scarring following SDM macular photocoagulation.

elevates the significance of associations if a high degree of dependency exists in the outcome between the two eyes of a patient. Our key findings, however, are descriptive in nature, and relate to visual acuity outcomes. Despite these cautions, the beneficial clinical results of SDM appear promising, especially since safety indicators such as adverse effects and laser scarring were not detected in any of the treated eyes. Prospective clinical trials are needed to confirm these findings and document their limitations.

\section{Authors' affiliations}

J K Luttrull, Private practice, Ventura, CA, USA

D C Musch, Departments of Ophthalmology and Visual Sciences and Epidemiology, Schools of Medicine and Public Health, University of Michigan, Ann Arbor, MI, USA

M A Mainster, Department of Ophthalmology, University of Kansas School of Medicine, Kansas City, Kansas

Commercial interests: JKL, none; DCM and MAM, consultants, Iridex, Mountain View, CA, USA.

\section{REFERENCES}

1 Kahn HA, Hiller R. Blindness caused by diabetic retinopathy. Am J Ophthalmol 1974;78:58-67.

2 Kahn HA, Bradley RF. Prevalence of diabetic retinopathy. Age, sex, and duration of diabetes. Br J Ophthalmol 1975;59:345-9.

3 Aiello LM, Rand LI, Briones JC, et al. Diabetic retinopathy in Joslin Clinic patients with adult-onset diabetes. Ophthalmology 1981;88:619-23.

4 Klein R, Klein BE, Moss SE, et al. The Wisconsin Epidemiologic Study of diabetic retinopathy. XIV. Ten-year incidence and progression of diabetic retinopathy. Arch Ophthalmol 1994;112:1217-28.

5 McMeel JW, Trempe CL, Franks EB. Diabetic maculopathy. Trans Am Acad Ophthalmol Otolaryngol 1977;83(Pt 1):OP476-87.

6 Bresnick GH. Diabetic macular edema. A review. Ophthalmology 1986;93:989-97

7 Early Treatment Diabetic Retinopathy Study Research Group Photocoagulation for diabetic macular edema. Early Treatment Diabetic Retinopathy Study report number 1. Arch Ophthalmol 1985;103:1796-806.
8 Schatz H, Madeira D, McDonald HR, et al. Progressive enlargement of laser scars following grid laser photocoagulation for diffuse diabetic macular edema. Arch Ophthalmol 1991;109:1549-51.

9 Morgan CM, Schatz H. Atrophic creep of the retinal pigment epithelium after focal macular photocoagulation. Ophthalmology 1989:96:96-103.

10 Early Treatment Diabetic Retinopathy Study Research Group. Focal photocoagulation treatment of diabetic macular edema. Relationship of treatment effect to fluorescein angiographic and other retinal characteristics at baseline: ETDRS report no 19. Arch Ophthalmol 1995;113:1144-55.

11 Lewen RM. Subretinal neovascularization complicating laser photocoagulation of diabetic maculopathy. Ophthalmic Surg 1988; 19:734-7.

12 Lewis H, Schachat AP, Haimann MH, et al. Choroidal neovascularization after laser photocoagulation for diabetic macular edema. Ophthalmology 1990;97:503-10, discussion 10-1.

13 Guyer DR, D'Amico DJ, Smith CW. Subretinal fibrosis after laser photocoagulation for diabetic macular edema. Am J Ophthalmol 1992;113:652-6.

14 Rutledge BK, Wallow IH, Poulsen GL. Sub-pigment epithelial membranes after photocoagulation for diabetic macular edema. Arch Ophthalmol 1993;111:608-13

15 Striph GG, Hart WM Jr, Olk RJ. Modified grid laser photocoagulation for diabetic macular edema. The effect on the central visual field. Ophthalmology 1988;95:1673-9.

16 Hudson C, Flanagan JG, Turner GS, et al. Influence of laser photocoagulation for clinically significant diabetic macular oedema (DMO) on short-wavelength and conventional automated perimetry. Diabetologia 1998;41:1283-92.

17 Ishiko S, Ogasawara H, Yoshida A, et al. The use of scanning laser ophthalmoscope microperimetry to detect visual impairment caused by macular photocoagulation. Ophthalmic Surg Lasers 1998;29:95-8.

18 Okuyama M, Okisaka S. Automatic static threshold perimetry is useful for estimating the effects of laser photocoagulation on diabetic maculopathy. Ophthalmic Res 1998;30:207-15.

19 Sinclair SH, Alaniz R, Presti P. Laser treatment of diabetic macular edema: comparison of ETDRS-level treatment with threshold-level treatment by using high-contrast discriminant central visual field testing. Sem Ophthalmol 1999; 14:214-22.

20 Mainster MA. Decreasing retinal photocoagulation damage: principles and techniques. Sem Ophthalmol 1999;14:200-9.

21 Jonas JB, Sofker A. Intraocular injection of crystalline cortisone as adjunctive treatment of diabetic macular edema. Am J Ophthalmol 2001;132:425-7.

22 Martidis A, Duker JS, Greenberg PB, et al. Intravitreal triamcinolone for refractory diabetic macular edema. Ophthalmology 2002;109:920-7.

23 Jonas JB, Kreissig I, Sofker A, et al. Intravitreal injection of triamcinolone for diffuse diabetic macular edema. Arch Ophthalmol 2003;121:57-61. 
24 Harbour JW, Smiddy WE, Flynn HW Jr, et al. Vitrectomy for diabetic macular edema associated with a thickened and taut posterior hyaloid membrane. Am J Ophthalmol 1996; 121:405-13.

25 La Heij EC, Hendrikse F, Kessels AG, et al. Vitrectomy results in diabetic macular oedema without evident vitreomacular traction. Graefes Arch Clin Exp Ophthalmol 2001;239:264-70.

26 Otani T, Kishi S. A controlled study of vitrectomy for diabetic macular edema. Am J Ophthalmol 2002;134:214-9.

27 Kojima T, Terasaki $\mathrm{H}$, Nomura $\mathrm{H}$, et al. Vitrectomy for diabetic macular edema: effect of glycemic control $(\mathrm{HbA}(1 \mathrm{c}))$, renal function (creatinine) and other local factors. Ophthalmic Res 2003;35:192-8.

28 Pankratov MM. Pulsed delivery of laser energy in experimental thermal retinal photocoagulation. Proc Soc Photo-Optical Instrum Eng 1990;1202:205-13.

29 Sliney DH, Marshall J. Tissue specific damage to the retinal pigment epithelium: mechanisms and therapeutic implications. Lasers Light Ophthalmol 1992;5:17-28

30 Roider J, Hillenkamp F, Flotte T, et al. Microphotocoagulation: selective effects of repetitive short laser pulses. Proc Natl Acad Sci USA 1993;90:8643-7.

31 Friberg TR, Karatza EC. The treatment of macular disease using a micropulsed and continuous wave 810-nm diode laser. Ophthalmology 1997; 104:2030-8.

32 Roider J, Brinkmann R, Wirbelaver C, et al. Retinal sparing by selective retinal pigment epithelial photocoagulation. Arch Ophthalmol 1999;117:1028-34.

33 Moorman CM. Hamilton AM. Clinical applications of the MicroPulse diode laser. Eye 1999;13(Pt 2):145-50.

34 Framme C, Brinkmann R, Birngruber R, et al. Autofluorescence imaging after selective RPE laser treatment in macular diseases and clinical outcome: a pilot study. Br J Ophthalmol 2002;86:1099-106.

35 Chew EY, Ferris FL 3rd, Csaky KG, et al. he long-term effects of laser photocoagulation treatment in patients with diabetic retinopathy: the early treatment diabetic retinopathy follow-up study. Ophthalmology 2003; 110:1683-9.

36 Olk RJ. Modified grid argon (blue-green) laser photocoagulation for diffuse diabetic macular edema. Ophthalmology 1986;93:938-50.

37 Olk RJ. Argon green (514 nm) versus krypton red (647 nm) modified grid laser photocoagulation for diffuse diabetic macular edema. Ophthalmology 1990;97:1101-12, discussion 12-13

38 Lee CM, Olk RJ. Modified grid laser photocoagulation for diffuse diabetic macular edema. Long-term visual results. Ophthalmology $1991 ; 98: 1594-602$

39 Akduman L, Olk RJ. Diode laser $(810 \mathrm{~nm})$ versus argon green $(514 \mathrm{~nm})$ modified grid photocoagulation for diffuse diabetic macular edema. Ophthalmology 1997;104:1433-41.

40 Akduman L, Olk RJ. Laser photocoagulation of diabetic macular edema. Ophthalmic Surg Lasers 1997;28:387-408.

41 Akduman L, Olk RJ. Subthreshold (subvisible) modified grid diode laser photocoagulation in diffuse diabetic macular edema (DDME). Ophthalmic Surg Lasers 1999;30:706-14.

42 Stanga PE, Reck AC, Hamilton AMP. Micropulse laser in the treatment of diabetic macular edema. Semin Ophthalmol 1999;14:210-3.

43 Roider J, Brinkmann R, Wirbelauer C, et al. Subthreshold (retinal pigment epithelium) photocoagulation in macular diseases: a pilot study. Br J Ophthalmol 2000;84:40-7.

44 Olk RJ, Akduman L. Minimal intensity diode laser (810 nanometer) photocoagulation (MIP) for diffuse diabetic macular edema (DDME). Sem Ophthalmol 2001;16:25-30.

45 Friberg TR. Infrared micropulsed laser treatment for diabetic macular edema-subthreshold versus threshold lesions. Sem Ophthalmol $2001 ; 16: 19-24$.

46 American National Standards Institute. American national standard for the safe use of lasers, ANSI Z136.1-2000. Washington, DC: American Nationa Standards Institute, 2000.

47 Sliney DH, Wolbarsht ML. Safety with lasers and other optical sources: a comprehensive handbook. New York: Plenum Press, 1980:1035.

48 Sliney DH, Mellerio J, Gabel VP, et al. What is the meaning of threshold in laser injury experiments? Implications for human exposure limits. Health Phys 2002:82:335-47.
49 Mainster MA, Turner PL. Retinal injuries from light: mechanisms, hazards and prevention. In: Ryan SJ, Ogden TE, Hinton DR, Schachat AP, eds. Retina. London: Elsevier Publishers, 2004:2

50 Treatment of Age-Related Macular Degeneration with Photodynamic Therapy (TAP) Study. Photodynamic therapy of subfoveal choroidal neovascularization in age- related macular degeneration with verteporfin: one-year results of 2 randomized clinical trials-TAP report. Group. Arch Ophthalmol 1999;117:1329-45.

51 Reichel E, Berrocal AM, Ip M, et al. Transpupillary thermotherapy of occult subfoveal choroidal neovascularization in patients with age-related macular degeneration. Ophthalmology 1999;106:1908-14.

52 Mainster MA, Reichel E. Transpupillary thermotherapy for age-related macular degeneration: long-pulse photocoagulation, apoptosis, and heat shock proteins. Ophthalmic Surg Lasers 2000;31:359-73.

53 Hillenkamp F. Interaction between laser radiation and biological systems. In: Hillenkamp F, Pratesi R, Sacchi CA, eds. Lasers in biology and medicine. New York: Plenum Publishing, 1980

54 Ham WT Jr, Mueller HA, Wolbarsht ML, et al. Evaluation of retinal exposures from repetitively pulsed and scanning lasers. Health Phys 1988;54:337-44.

55 Mainster MA, White TJ, Tips JH, et al. Retinal-temperature increases produced by intense light sources. J Opt Soc Am 1970;60:264-70.

56 Birngruber R. Thermal modeling in biological tissues. In: Hillenkamp F, Pratesi R, Sacchi CA, eds. Lasers in medicine and biology. New York: Plenum Publishing, 1980

57 Hogan MJ, Alvarado JA, Weddell JE. Histology of the human eye: an atlas and textbook. Philadelphia: WB Saunders, 1971

58 Fine BS, Yanoff M. Ocular histology: a text and atlas. New York: Harper and Row, 1979

59 Bresnick GH. Diabetic maculopathy. A critical review highlighting diffuse macular edema. Ophthalmology 1983;90:1301-17.

60 Aiello LP, Avery RL, Arrigg PG, et al. Vascular endothelial growth factor in ocular fluid of patients with diabetic retinopathy and other retinal disorders. N Engl J Med 1994:331:1480-7.

61 Antonetti DA, Lieth E, Barber AJ, et al. Molecular mechanisms of vascular permeability in diabetic retinopathy. Sem Ophthalmol 1999;14:240-8.

62 Mainster MA, Warren KA. Retinal photocoagulation. In: Guyer DR, Yannuzzi LA, Chang S, et al, eds. Retina-vitreous-macula. New York: WB Saunders, 1999; 1 .

63 Stefansson E. The therapeutic effects of retinal laser treatment and vitrectomy. A theory based on oxygen and vascular physiology. Acta Ophthalmol Scand $2001 ; 79: 435-40$.

64 Ogata N, Wang L, Jo N, et al. Pigment epithelium derived factor as a neuroprotective agent against ischemic retinal injury. Curr Eye Res $2001 ; 22: 245-52$

65 Mori K, Gehlbach P, Ando A, et al. Regression of ocular neovascularization in response to increased expression of pigment epithelium-derived factor. Invest Ophthalmol Vis Sci 2002;43:2428-34.

66 Wilson AS, Hobbs BG, Shen WY, et al. Argon laser photocoagulationinduced modification of gene expression in the retina. Invest Ophthalmol Vis Sci 2003;44:1426-34

67 Desmettre T, Maurage CA, Mordon S. Transpupillary thermotherapy (TT) with short duration laser exposures induce heat shock protein (HSP) hyperexpression on choroidoretinal layers. Lasers Surg Med 2003;33:102-7.

68 Marshall J, Bird AC. A comparative histopathological study of argon and krypton laser irradiations of the human retina. Br J Ophthalmol 1979:63:657-68.

69 Wallow IH, Bindley CD. Focal photocoagulation of diabetic macular edema. A clinicopathologic case report. Retina 1988;8:261-9.

70 Bandello F, Brancato R, Menchini U, et al. Light panretinal photocoagulation (LPRP) versus classic panretinal photocoagulation (CPRP) in proliferative diabetic retinopathy. Sem Ophthalmol 2001;16:12-8

71 Roider J, Brinkmann R, Framme C, et al. Selective RPE Laser Treatment in Macular Diseases: Clinical results. Invest Ophthalmol Vis Sci 2001;42:S695.

72 Ferris F. Early photocoagulation in patients with either type I or type II diabetes. Trans Am Ophthalmol Soc 1996:94:505-37.

73 Aiello LM. Perspectives on diabetic retinopathy. Am J Ophthalmo 2003;136:122-35. 